\title{
Attitude Dynamics and Stability of a Simple Solar Photon Thruster
}

\author{
Anna D. Guerman, ${ }^{1}$ Georgi Smirnov, ${ }^{2}$ and Maria Cecilia Pereira ${ }^{3}$ \\ ${ }^{1}$ Centre for Mechanical and Aerospace Science and Technologies, University of Beira Interior, 6201-001 Covilhã, Portugal \\ ${ }^{2}$ Centre of Physics, Department of Mathematics and Applications, University of Minho, Campus de Gualtar, 4710-057 Braga, Portugal \\ ${ }^{3}$ Department of Mechanical Engineering, Federal University of Minas Gerais, 31270-901 Belo Horizonte, MG, Brazil
}

Correspondence should be addressed to Anna D. Guerman; anna@ubi.pt

Received 21 July 2013; Accepted 9 September 2013

Academic Editor: Antonio F. Bertachini A. Prado

Copyright (C) 2013 Anna D. Guerman et al. This is an open access article distributed under the Creative Commons Attribution License, which permits unrestricted use, distribution, and reproduction in any medium, provided the original work is properly cited.

This paper is dedicated to the development of a model of the attitude dynamics for a nonideal Simple Solar Photon Thruster (SSPT) and to the analysis of sailcraft motions with respect to their centre of mass. Derivation of the expressions for force and torque due to solar radiation that is valid for the case, when there is a misalignment of the SSPT axis with the sun direction, is followed by study of sailcraft dynamics and stability properties. Analysis of stability shows that an ideally reflecting sail is unstable, while for a sailcraft with nonideal collector, the symmetry axis is stable with respect to the Sun direction for large variety of system parameters. The motion around symmetry axis is always unstable and requires an active stabilizer.

\section{Introduction}

Solar sails are attractive spacecrafts for long missions, for they create a propulsion effort using freely available solar radiation pressure. The initial concepts of such vehicle have been proposed in the 1920s $[1,2]$ as an alternative for minimizing on-board energy consumption. With the recent development of new films, materials, and structures its implementation has become possible making solar sails a subject of detailed research regarding eventual missions, the respective control issues, the sailcraft structure, lab testing, and so forth (see, e.g., [3-12], just to name a few), and leading to the implementation of the first sailcraft mission by JAXA in 2010 [10].

The large majority of these studies are focused on the analysis of the Flat Solar Sail (FSS); its principal element is a large flat surface formed by a reflecting membrane. Control of FSS orbital motion is performed by turning the entire sail with respect to the sunlight direction. As a result, the radiation pressure on the surface changes, enabling the control of the vehicle trajectory. Attitude dynamics of such sailcraft are studied, for example, in $[4,11,12]$.
Another possible scheme for a sailcraft is the Solar Photon Thruster (SPT). Its main idea is to separate the functions of collecting and reflecting the incoming light. Even though the SSPT's general scheme has been proposed long ago $[1,2,13]$, the detailed research has begun quite recently [14-22]. SPT consists of a parabolic reflecting surface (collector) that is always pointing towards the sun, taking advantage of the totality of the sail area. The incoming light is then reflected on a system of smaller mirrors capable of controling the direction of the outgoing light; the sailcraft is, therefore, controlled more easily by the rotation of one smaller mirror.

The SPT can be either Simple SPT (SSPT) or Dual Reflector SPT (DR SPT) depending on the system of smaller mirrors used. The SSPT is provided with two main elements, the Collector C and the Director mirror D $[1,13-15,18$, 19]. Ideally, the symmetry axis of the collector $C$ remains aligned to the sun-sailcraft direction, and all incoming light is concentrated on the collector focus. Director D is placed at this focus; it can rotate changing the direction of the outgoing light in order to control the force acting on the sailcraft and therefore its motion. 


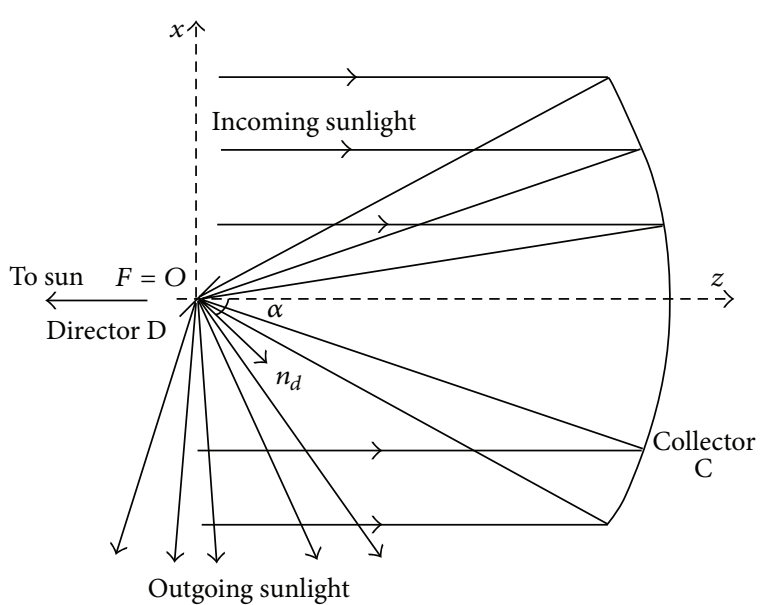

FIgure 1: Scheme of a Simple Solar Photon Thruster.

Several versions of DR SPT structure (see, e.g., $[1,13,19-$ 21]) include one more parabolic mirror, called Reflector $R$. The incoming light from $\mathrm{C}$ hits $\mathrm{R}$, becoming a parallel beam that is reflected on $\mathrm{D}$. The director can rotate controlling the resulting propulsion.

Previous studies [17, 19] show that SSPT has many advantages compared to DR SPT. Although its heat tolerance requirements are higher than DR SPT (all the incoming light is concentrated at one point of the director), the advantages are more numerous. When compared to DR SPT, SSPT is much simpler, being therefore easier to deploy and maintain during the flight, and more compact and lighter, thus saving space for the payload. SSPT is more robust than DR SPT regarding the deformations of the collector shape and misalignments with respect to sun-sailcraft direction. For the above reasons, SSPT has been chosen to be the subject of more detailed studies.

In [22], the first model of force acting on SSPT which is assumed to be perfectly aligned with the sun-sailcraft direction is presented and some trajectory simulations are performed. Here, elaboration of a force and torque model for SSPT attending to the eventual axis misalignment permits one to study the sailcraft attitude dynamics and stability properties.

\section{Force and Torque Model for a Nonideal Sailcraft}

Figure 1 shows the general scheme of SSPT. The Collector C is a big parabolic reflecting surface which concentrates the parallel beam of the incoming sunlight at its focus F. Director $\mathrm{D}$ is a relatively small plane mirror located at the focus of the collector. The force acting on the sail is controlled by rotating $\mathrm{D}$ which changes the direction of outgoing light varying the magnitude and direction of the total propulsion force.

In [22], the general characteristics of a compound solar sail have been analysed. The results show that for minimizing collector film overheating in order to lower the thermal requirements, the light beam should not reflect on the

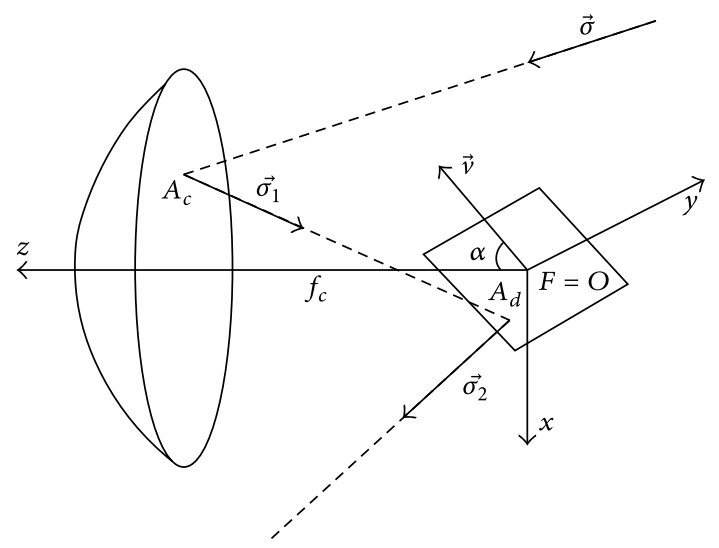

FIGURE 2: Scheme of reflections on SSPT with axis misalignment.

collector more than once. To avoid secondary reflections, the angle $\widetilde{\alpha}$ between the collector's symmetry axis and the normal to the director's surface must satisfy the following condition:

$$
\tan \tilde{\alpha}>\frac{4 f_{c} R_{c}}{\left(4 f_{c}^{2}-R_{c}^{2}\right)} .
$$

Here, $f_{c}$ and $R_{c}$ are the focal distance and the effective radius of the collector, respectively. If the control angle is limited by condition (1), the components of the force and torque due to solar radiation pressure can be obtained analytically.

A force and torque model for SSPT has been developed in [22] assuming that all sailcraft elements possess ideally reflecting surfaces. But as the collector is a large surface, it should be made of a thin film and it cannot be an ideal reflector. The director is much smaller, so it can have better optical properties. So, here a model for force and torque considering a nonideal collector is developed. The director is supposed to be ideal and small enough so that the effect of its shadow can be disregarded. Consider a SSPT whose symmetry axis is misaligned in relation to sunlight direction.

To calculate the vectors of force and torque due to solar radiation on the SSPT, consider a parabolic surface (collector) shown in Figure 2. Its equation in the reference frame $O x y z$, in which the center of mass $O$ coincides with the focus $F$ of the paraboloid and $\mathrm{O} z$ is its symmetry axis, is given by

$$
x^{2}+y^{2}+4 f_{c}\left(z-f_{c}\right)=0
$$

or

$$
z=\frac{1}{4 f_{c}}\left(4 f_{c}-x^{2}-y^{2}\right)=0
$$

Suppose that the incoming sunlight has the direction $\vec{\sigma}$ (Figure 2). It is absorbed by the collector at the point $A_{c}$ of the element of the parabolic surface $d S$ with the position vector $\vec{\xi}=x \vec{i}+y \vec{j}+z \vec{k}$, producing the force

$$
d \vec{P}_{1}=-\rho \vec{\sigma}(\vec{\sigma} \cdot \vec{n}) d S
$$


where $\rho=\Phi r_{E}^{2} / r_{s}^{2}$ is the intensity of the light flow at the current point of the orbit. The respective torque about the center of the mass of the SSPT $O$ is

$$
d \vec{M}_{1}=\vec{\xi} \times d \vec{P}_{1} .
$$

A fraction $\tilde{r}$ of the incident light is reflected on the element of the surface $d S$, and a fraction $s$ of the reflected light is reflected specularly, while a fraction $(1-s)$ is scattered from the collector surface in a nonspecular way [1]. After hitting the collector, specularly reflected light has direction $\vec{\sigma}_{1}$ as follows:

$$
\vec{\sigma}_{1}=\vec{\sigma}-2(\vec{\sigma} \cdot \vec{n}) \vec{n},
$$

where $\vec{n}$ is the internal normal unit vector to the surface at point $\vec{\xi}$ given by

$$
\vec{n}=-\frac{x \vec{i}+y \vec{j}+2 f_{c} \vec{k}}{\sqrt{x^{2}+y^{2}+4 f_{c}^{2}}} .
$$

In (7), $\vec{i}, \vec{j}$, and $\vec{k}$ are the Oxyz coordinate orts. This fraction of light that is reflected specularly generates a force

$$
d \vec{P}_{2 r}=\rho \widetilde{r} s \vec{\sigma}_{1}(\vec{\sigma}, \vec{n}) d S,
$$

while the nonspecularly reflected light generates a force given by

$$
d \vec{P}_{2 s}=\rho B_{f}(1-s) \vec{r} \vec{n}(\vec{\sigma}, \vec{n}) d S .
$$

Moreover, a portion of the absorbed light is reemitted backwards creating the force

$$
d \vec{P}_{2 e}=\rho(1-\widetilde{r}) \frac{\varepsilon_{f} B_{f}-\varepsilon_{b} B_{b}}{\varepsilon_{f}+\varepsilon_{b}} \vec{n}(\vec{\sigma}, \vec{n}) d S .
$$

In (10), $B_{f}$ and $B_{b}$ are the surface front and back nonLambertian coefficients and $\varepsilon_{f}$ and $\varepsilon_{b}$ are the front and back emissivities. The total force applied is described as

$$
d \vec{P}_{2}=d \vec{P}_{2 r}+d \vec{P}_{2 s}+d \vec{P}_{2 e} .
$$

It produces the torque with respect to the center of mass $O$ given by

$$
d \vec{M}_{2}=\vec{\xi} \times d \vec{P}_{2} .
$$

Then, the light is reflected by the director whose orientation can be described by the normal to its surface $\vec{v}$. One can assume that the light hitting D is only the fraction specularly reflected on the collector: as the director is small compared to the collector, the respective spatial angle observed from the collector is small and the influence of the scattered light may be disregarded. The incoming light from point $A_{c}$ hits the director at point $A_{d}$ with the position vector that can be found from condition (13) as follows:

$$
0=\left(\vec{\xi}_{A_{d}} \cdot \vec{\nu}\right)=\left(\vec{\xi}+\tau \vec{\sigma}_{1} \cdot \vec{\nu}\right),
$$

so,

$$
\begin{gathered}
\tau=-\frac{(\vec{\xi} \cdot \vec{v})}{\left(\vec{\sigma}_{1} \cdot \vec{v}\right)}, \\
\vec{\xi}_{A_{d}}=\vec{\xi}-\frac{(\vec{\xi} \cdot \vec{v})}{\left(\vec{\sigma}_{1} \cdot \vec{v}\right)} \vec{\sigma}_{1} .
\end{gathered}
$$

Force and torque transmitted by the light hitting the director are, respectively, as follows:

$$
\begin{gathered}
d \vec{P}_{3}=-\rho \widetilde{r} s \vec{\sigma}_{1}(\vec{\sigma} \cdot \vec{n}) d S, \\
d \vec{M}_{3}=\vec{\xi}_{A_{d}} \times d \vec{P}_{3}=\rho \widetilde{r} s\left(\vec{\sigma}_{1} \times \vec{\xi}\right)(\vec{\sigma} \cdot \vec{n}) d S .
\end{gathered}
$$

The reflection of the light at the director produces force and torque that can be written, respectively, as

$$
\begin{gathered}
d \vec{P}_{4}=\rho \widetilde{r} s \vec{\sigma}_{2}(\vec{\sigma} \cdot \vec{n}) d S, \\
d \vec{M}_{4}=\vec{\xi}_{A_{d}} \times d \vec{P}_{4},
\end{gathered}
$$

where

$$
\vec{\sigma}_{2}=\vec{\sigma}_{1}-2\left(\vec{\sigma}_{1} \cdot \vec{v}\right) \vec{\nu}
$$

is the direction of the light reflected on the control mirror D.

The interaction of the light beam caught by the element $d S$ of the collector $\mathrm{C}$ with the sail structure generates the following elementary force:

$$
d \vec{P}=d \vec{P}_{1}+d \vec{P}_{2}+d \vec{P}_{3}+d \vec{P}_{4}
$$

The total force actuating on the sailcraft due to sunlight radiation pressure can be obtained by integration as follows:

$$
\vec{P}=\int_{C} d \vec{P}
$$

After integration, one gets

$$
\begin{aligned}
& P_{x}=\pi \rho\left[\chi_{x}+\zeta_{x} \ln \left(1+\frac{R_{c}^{2}}{4 f_{c}^{2}}\right)\right], \\
& P_{y}=\pi \rho\left[\chi_{y}+\zeta_{y} \ln \left(1+\frac{R_{c}^{2}}{4 f_{c}^{2}}\right)\right], \\
& P_{z}=\pi \rho\left[\chi_{z}+\zeta_{z} \ln \left(1+\frac{R_{c}^{2}}{4 f_{c}^{2}}\right)\right] .
\end{aligned}
$$


Here,

$$
\begin{aligned}
& \chi_{x}=\frac{1}{6 f_{c}}\left\{\sigma_{x} \lambda \Psi-6 f_{c} R_{c}^{2}\right. \\
& \times\left[-\sigma_{x} \sigma_{z}\right. \\
& +\widetilde{r} s\left(-\sigma_{x} \sigma_{z}\left(1-2 v_{x}^{2}\right)\right. \\
& \left.\left.\left.+2 v_{x}\left(v_{z}+v_{y} \sigma_{y} \sigma_{z}-2 v_{z} \sigma_{z}^{2}\right)\right)\right]\right\}, \\
& \chi_{y}=\frac{1}{6 f_{c}}\left\{\sigma_{y} \lambda \Psi-6 f_{c} R_{c}^{2}\right. \\
& \times\left[-\sigma_{y} \sigma_{z}\right. \\
& +\widetilde{r} s\left(-\sigma_{y} \sigma_{z}\left(1-2 v_{y}^{2}\right)\right. \\
& \left.\left.\left.+2 v_{y}\left(v_{z}+v_{x} \sigma_{x} \sigma_{z}-2 v_{z} \sigma_{z}^{2}\right)\right)\right]\right\}, \\
& \chi_{z}=4 f_{c} \sigma_{z}\left(-2 f_{c}+\sqrt{R_{c}^{2}+4 f_{c}^{2}}\right) \Psi \\
& +R_{c}^{2}\left[\sigma_{z}^{2}+\widetilde{r} s\left(1-\sigma_{z}^{2}\left(1-2 v_{z}^{2}\right)\right.\right. \\
& \left.\left.-2 v_{z}\left(v_{z}+v_{x} \sigma_{x} \sigma_{z}+v_{y} \sigma_{y} \sigma_{z}\right)\right)\right],
\end{aligned}
$$

with

$$
\begin{gathered}
\lambda=16 f_{c}^{3}+\left(R_{c}^{2}-8 f_{c}^{2}\right) \sqrt{R_{c}^{2}+4 f_{c}^{2}}, \\
\Psi=\frac{B_{b} \varepsilon_{b}(\widetilde{r}-1)+B_{f}\left[\varepsilon_{f}+\widetilde{r} \varepsilon_{b}-\widetilde{r} s\left(\varepsilon_{f}+\varepsilon_{b}\right)\right]}{\varepsilon_{f}+\varepsilon_{b}}, \\
\zeta_{x}=8 f_{c}^{2} \widetilde{r} s\left[-\sigma_{x} \sigma_{z}\left(1-2 v_{x}^{2}\right)\right. \\
\left.+v_{x}\left(v_{z}+2 v_{y} \sigma_{y} \sigma_{z}-3 v_{z} \sigma_{z}^{2}\right)\right], \\
\zeta_{y}=8 f_{c}^{2} \widetilde{r} s\left[-\sigma_{y} \sigma_{z}\left(1-2 v_{y}^{2}\right)\right. \\
\left.+v_{y}\left(v_{z}+2 v_{x} \sigma_{x} \sigma_{z}-3 v_{z} \sigma_{z}^{2}\right)\right], \\
\zeta_{z}=4 f_{c}^{2} \widetilde{r} s\left[1-3 \sigma_{z}^{2}\left(1-2 v_{z}^{2}\right)\right. \\
\left.-2 v_{z}\left(v_{z}+2 v_{x} \sigma_{x} \sigma_{z}+2 v_{y} \sigma_{y} \sigma_{z}\right)\right] .
\end{gathered}
$$

For the case when the ideally reflecting SSPT is completely aligned with the sun direction $\left(\sigma_{x}=\sigma_{y}=0\right.$ and $\left.\sigma_{z}=1\right)$ and the director rotates in the plane of the orbit $\left(v_{x}=\sin \widetilde{\alpha}, v_{y}=0\right.$, and $\left.v_{z}=\cos \widetilde{\alpha}\right)$, expressions (20) are reduced to

$$
\begin{gathered}
P_{X}=\frac{\Phi r_{E}^{2}}{r_{s}^{2}} \pi R_{c}^{2}\left[1-8 \frac{f_{c}^{2}}{R_{c}^{2}} \ln \left(1+\frac{R_{c}^{2}}{4 f_{c}^{2}}\right)\right] \sin 2 \widetilde{\alpha}, \\
P_{Y}=0, \\
P_{Z}=2 \frac{\Phi r_{E}^{2}}{r_{s}^{2}} \pi R_{c}^{2}\left[\cos ^{2} \widetilde{\alpha}-4 \frac{f_{c}^{2}}{R_{c}^{2}} \ln \left(1+\frac{R_{c}^{2}}{4 f_{c}^{2}}\right) \cos 2 \widetilde{\alpha}\right] .
\end{gathered}
$$

These expressions for the solar radiation force acting on SSPT have been used previously to study orbital transfer problems for SSPT and FSS [16, 22]. These studies show several advantages of SSPT scheme compared to FSS that are more evident for large-scale missions (i.e., for Solar System escape).

Calculation of the total torque due to the light pressure results in the following expressions:

$$
\begin{aligned}
& M_{s x}=\pi \rho\left\{\frac{\sigma_{y} v \Psi}{120 f_{c}^{2}}\right. \\
&+ \frac{R_{c}^{2} \sigma_{z}}{4 f_{c}}\left[\left(R_{c}^{2}-4 f_{c}^{2}\right) \sigma_{y}\right. \\
&\left.\left.-4 f_{c}^{2} \widetilde{r} s\left(2 v_{x} v_{y} \sigma_{x}+\left(1-2 v_{x}^{2}\right) \sigma_{y}\right)\right]\right\}, \\
& M_{s y}=\pi \rho\left\{-\frac{\sigma_{x} v \Psi}{120 f_{c}^{2}}\right.+\frac{R_{c}^{2} \sigma_{z}}{4 f_{c}}\left[-\left(R_{c}^{2}-4 f_{c}^{2}\right) \sigma_{x}\right. \\
&\left.\left.+4 f_{c}^{2} \widetilde{r} s\left(2 v_{x} v_{y} \sigma_{y}+\left(1-2 v_{y}^{2}\right) \sigma_{x}\right)\right]\right\}, \\
& M_{s z}=2 \pi \rho f_{c} R_{c}^{2} \widetilde{r} s v_{z} \sigma_{z}\left(v_{x} \sigma_{y}-v_{y} \sigma_{x}\right),
\end{aligned}
$$

where $\Psi$ is defined in (22) and

$$
v=64 f_{c}^{5}+\left(3 R_{c}^{4}+4 f_{c}^{2} R_{c}^{2}-32 f_{c}^{4}\right) \sqrt{R_{c}^{2}+4 f_{c}^{2}} .
$$

These models for force and torque are used later on to study the attitude dynamics of SSPT.

\section{SSPT Attitude Dynamics}

Consider the problem of a SSPT attitude dynamics on a heliocentric orbit. This analysis is done using two right Cartesian reference frames located at the SSPT center of mass $O . O x y z$ is the reference frame connected with the sailcraft; its axes are SSPT central principle axes of inertia. OXYZ is the frame related to the orbital motion of the SSPT; its unit vectors along the axes are

$$
\begin{gathered}
\vec{e}_{Z}=\frac{\vec{r}_{O}}{r_{O}}, \\
\vec{e}_{Y}=\vec{e}_{Z} \times \frac{\vec{v}_{O}}{v_{O}}, \\
\vec{e}_{X}=\vec{e}_{Y} \times \vec{e}_{Z},
\end{gathered}
$$

where $\vec{r}_{O}$ is the radius vector of point $O$ with respect to the center of the sun and $\vec{v}_{O}$ is SSPT's center of mass velocity. Obviously, the sunlight follows in the direction of $\mathrm{OZ}$ axis.

Mutual orientation of these frames can be described by the orthogonal matrix $A=\left[a_{i j}\right]$, where the elements $a_{i j}$ are 
the direction cosines of the axes of the $O x y z$ system with respect to $O X Y Z$. The transition from system $O X_{1} X_{2} X_{3}$ to system $O x_{1} x_{2} x_{3}$ can be done by three Euler's rotations about axes 1,2 , and 3 through the angles $\gamma$ (roll), $\alpha$ (pitch), and $\beta$ (yaw). The direction cosines $a_{i j}$ are expressed through these angles as

$$
\begin{gathered}
a_{11}=\cos \left(x_{1}, X_{1}\right)=\cos \alpha \cos \beta, \\
a_{12}=\cos \left(x_{2}, X_{1}\right)=-\cos \alpha \sin \beta, \\
a_{13}=\cos \left(x_{3}, X_{1}\right)=\sin \alpha, \\
a_{21}=\cos \left(x_{1}, X_{2}\right)=\sin \beta \cos \gamma+\sin \alpha \cos \beta \sin \gamma, \\
a_{22}=\cos \left(x_{2}, X_{2}\right)=\cos \beta \cos \gamma-\sin \alpha \sin \beta \sin \gamma, \\
a_{23}=\cos \left(x_{3}, X_{2}\right)=-\cos \alpha \sin \gamma, \\
a_{31}=\cos \left(x_{1}, X_{3}\right)=\sin \beta \sin \gamma-\sin \alpha \cos \beta \cos \gamma, \\
a_{32}=\cos \left(x_{2}, X_{3}\right)=\cos \beta \sin \gamma+\sin \alpha \sin \beta \cos \gamma, \\
a_{33}=\cos \left(x_{3}, X_{3}\right)=\cos \alpha \cos \gamma .
\end{gathered}
$$

At the heliocentric trajectories, the characteristic values of the angular acceleration due to orbital motion and gravitygradient torque are much smaller than those created by $\mathrm{cm}-\mathrm{cp}$ offset. Taking into account only the latter effect, the equations of attitude motion can be written as

$$
\begin{aligned}
& A \dot{p}+(C-B) q r=M_{s x}, \\
& B \dot{q}+(A-C) r p=M_{s y}, \\
& C \dot{r}+(B-A) p q=M_{s z},
\end{aligned}
$$

where $A, B$, and $C$ are the principal moments of inertia of the sailcraft and $M_{s x}, M_{s y}$, and $M_{s z}$ are the components of the solar radiation torque given by (24). The projections of the sailcraft absolute angular velocity onto the axes $O x y z, p, q$, and $r$ can be expressed as

$$
\begin{gathered}
p=\dot{\gamma} a_{11}+\dot{\alpha} \sin \beta, \\
q=\dot{\gamma} a_{12}+\dot{\alpha} \cos \beta, \\
r=\dot{\gamma} a_{13}+\dot{\beta} .
\end{gathered}
$$

The direction of sunlight is $\vec{\sigma}=\left(a_{31}, a_{32}, a_{33}\right)$. Equations of motion (28) and (29) admit the following trivial solution:

$$
\alpha=\beta=\gamma=0 \text {. }
$$

Solution (30) corresponds to the nominal equilibrium orientation. According to the SSPT force and torque model, the solar radiation torque acting on an SPT in the vicinity of this equilibrium has the components

$$
\begin{gathered}
M_{s x}=G v_{x} v_{y} \alpha+\left(F+v_{x}^{2} G\right) \gamma, \\
M_{s y}=\left(F+v_{y}^{2} G\right) \alpha+G v_{x} \nu_{y} \gamma, \\
M_{s z}=G v_{z}\left(\nu_{y} \alpha+v_{x} \gamma\right),
\end{gathered}
$$

where

$$
\begin{gathered}
F=\pi \rho\left[\frac{v \Psi}{120 f_{c}^{2}}+\frac{R_{c}^{2}}{4 f_{c}}\left(R_{c}^{2}-4 f_{c}^{2}\right)-R_{c}^{2} f_{c} \tilde{r} s\right], \\
G=2 \pi \rho R_{c}^{2} f_{c} \tilde{r} s .
\end{gathered}
$$

Assuming that the attitude motion of SSPT occurs in the vicinity of the equilibrium orientation, (28) and (29) can be linearized, resulting in

$$
\begin{gathered}
B \ddot{\alpha}-\left(F+\nu_{y}^{2} G\right) \alpha-G v_{x} \nu_{y} \gamma=0, \\
A \ddot{\gamma}-G v_{x} \nu_{y} \alpha-\left(F+v_{x}^{2} G\right) \gamma=0, \\
C \ddot{\beta}=G v_{z}\left(\nu_{y} \alpha+v_{x} \gamma\right) .
\end{gathered}
$$

Analyzing linearized system (33), one can notice that the motion of the $\mathrm{O} z$ axis does not depend on the yaw angle $\beta$. The rotation of the sailcraft about the collector symmetry axis is unstable. Considering that the sailcraft is an axisymmetric body, that is, $A=B$, the stability conditions for motion of the $\mathrm{O} z$ axis can be written using the characteristic equation

$$
\begin{aligned}
A^{2} \lambda^{4}-A\left[2 F+G\left(v_{x}^{2}+v_{y}^{2}\right)\right] \lambda^{2} \\
+F\left[F+G\left(v_{x}^{2}+v_{y}^{2}\right)\right]=0 .
\end{aligned}
$$

For stability of zero equilibrium position of system (33), characteristic equation (34) should have purely imaginary roots, which happens if and only if

$$
\begin{gathered}
2 F+G\left(v_{x}^{2}+v_{y}^{2}\right)<0, \\
F\left[F+G\left(v_{x}^{2}+v_{y}^{2}\right)\right]>0, \\
{\left[2 F+G\left(v_{x}^{2}+v_{y}^{2}\right)\right]^{2}-4 F\left[F+G\left(v_{x}^{2}+v_{y}^{2}\right)\right]>0 .}
\end{gathered}
$$

As one can see, $G>0$, and so conditions (35) are equivalent to

$$
F<-G\left(v_{x}^{2}+v_{y}^{2}\right)
$$

$\mathrm{O} z$ axis should be stable for all possible orientations of the director. Considering that director rotation is limited by condition (1), the components of the normal to the director surface $v_{x}$ and $v_{y}$ must be limited by

$$
\frac{16 f_{c}^{2} R_{c}^{2}}{\left(4 f_{c}^{2}+R_{c}^{2}\right)^{2}}<v_{x}^{2}+v_{y}^{2} \leq 1 .
$$

This condition is satisfied for all possible values of $v_{x}$ and $v_{y}$ if and only if

$$
F<-G \text {. }
$$


Writing condition (38) in terms of the physical characteristics of the collector film, one gets

$$
\begin{aligned}
& 15 \psi(\widetilde{r} s+\psi-1) \\
& \quad+\frac{1}{\varepsilon_{b}+\varepsilon_{f}}\left[B_{b} \varepsilon_{b}(\tilde{r}-1)+B_{f}\left(\varepsilon_{f}+\widetilde{r} \varepsilon_{b}-s \widetilde{r}_{f} \varepsilon_{b}-s \widetilde{r}_{f} \varepsilon_{f}\right)\right] \\
& \quad \times\left[2+(3 \psi-2)(1+\psi)^{3 / 2}\right]<0,
\end{aligned}
$$

where $\psi=\left(R_{c} / 2 f_{c}\right)^{2}$.

3.1. Stability of a Solar Sail with Ideal Collector. For a sailcraft with an ideal collector, the surface optical parameters are [1] as follows:

$$
\tilde{r}=1, \quad s=1, \quad \varepsilon_{b}=\varepsilon_{f}, \quad B_{b}=B_{f}=\frac{2}{3} .
$$

In this case, condition (39) becomes

$$
4 f_{c}^{3} \pi \rho \psi^{2}<0
$$

So, for all geometries of the sail, the $\mathrm{O} z$ axis is unstable.

3.2. Stability of a Solar Sail with Black Collector. For a sailcraft with an ideally black film in collector, the surface optical parameters are [1]

$$
\tilde{r}=0, \quad s=0, \quad B_{b}=B_{f}=0 .
$$

In this case, condition (39) becomes

$$
4 f_{c}^{3} \pi \rho \psi(\psi-1)<0
$$

Condition (43) means that for an ideal black collector the motion of the $\mathrm{O} z$ axis is stable for $\psi<1$. So, for black sails with $R_{c}<2 f_{c}$, SSPT axis is stable.

3.3. Stability of a General Solar Sail. For sailcraft with general properties of the film, condition (39) holds for some $0<\psi<$ $\psi_{0}$. Considering the case described in [1], the film properties are

$$
\begin{aligned}
& \tilde{r}=0.88, \quad s=0.94, \quad \varepsilon_{b}=0.55 \\
& \varepsilon_{f}=0.05, \quad B_{b}=0.55, \quad B_{f}=0.79 .
\end{aligned}
$$

Condition (39) for this case means that the sailcraft $\mathrm{Oz}$ axis is stable if $R_{c}<0.83 f_{c}$.

The stability of $\mathrm{O} z$ axis does not mean that the sailcraft is stable. Its motion about $\mathrm{Oz}$ axis is always unstable, being a combination of harmonic oscillation and rotation with constant angular velocity. Meanwhile, the orientation of a SSPT about $\mathrm{O} z$ axis should be stabilized since it determines the director position, so a stabilizer for $\beta$ is always necessary.

\section{Conclusion}

In this paper, a new model for force and torque actuating on a compound solar sail is developed and used to study sailcraft motions. The attitude dynamics of a SSPT is studied for the case when the collector film possesses nonideal optical properties and the sailcraft symmetry axis is not aligned to the sun-sailcraft direction. Analysing the sailcraft stability, one can see that the solar radiation pressure destabilizes an ideal sail, but non-ideal collector film contributes to stabilization, resulting in stability, of its symmetry axis for all orientations of director when collector focal distance is large enough. However, an active control system is always necessary to guarantee stabilization of the sailcraft orientation with respect to the collector's symmetry axis.

\section{Conflict of Interests}

The authors declare that they have no conflict of interests.

\section{Acknowledgments}

This research is supported by the Brazilian program "Science without Borders" and Project ODySSea (PTDC/CTESPA/ 098030/2008) funded by the Portuguese Foundation for Science and Technologies (FCT), the Portuguese Operational Program for Competitiveness Factors (COMPETE), the Portuguese Strategic Reference Framework (QREN), and the European Regional Development Fund (FEDER).

\section{References}

[1] C. R. McInnes, Solar Sailing: Technology, Dynamics and Mission Applications, Springer, Berlin, Germany, 1999.

[2] E. N. Polyahova, Space Flight With Solar Sail, 'Nauka', Moscow, Russia, 1986.

[3] M. MacDonald and C. McInnes, "Solar sail science mission applications and advancement," Advances in Space Research, vol. 48, no. 11, pp. 1702-1716, 2011.

[4] B. Wie, "Solar sail attitude control and dynamics," Journal of Guidance, Control, and Dynamics, vol. 27, no. 4, pp. 536-544, 2004.

[5] D. W. Sleight and D. M. Muheim, "Parametric studies of square solar sails using finite element analysis," in Proceedings of the 45th AIAA/ASME/ASCE/AHS Structures, Structural Dynamics and Materials Conference, pp. 85-97, Palm Springs, California, April 2004.

[6] Z. G. Jinjin, S. Zhaoyu, Z. Hongjun, H. Wenhao, and S. Lianguan, "Structural analysis about a new solar sail," Chinese Journal of Space Science, no. 2, pp. 249-256, 2009.

[7] Q. Li, X. Ma, and T. Wang, "Reduced model for flexible solar sail dynamics," Journal of Spacecraft and Rockets, vol. 48, no. 3, pp. 446-453, 2011.

[8] F. Couceiro, P. Gamboa, J. M. Silva, and A. D. Guerman, "Membrane-based space energy collector: a conceptual study," Acta Astronautica, vol. 87, pp. 8-13, 2013.

[9] B. K. Taleghani, P. S. Lively, J. L. Gaspar, D. M. Murphy, and T. A. Trautt, "Dynamic and static shape test/analysis correlation of a 10 meter quadrant solar sail," in Proceedings of the 46th AIAA/ASME/ASCE/AHS/ASC Structures, Structural Dynamics 
and Materials Conference, pp. 4003-4016, Austin, Tex, USA, April 2005.

[10] Y. Tsuda, O. Mori, R. Funase et al., "Achievement of IKAROSJapanese deep space solar sail demonstration mission," Acta Astronautica, vol. 82, no. 2, pp. 183-188, 2013.

[11] Z. Jin and W. Tianshu, "Coupled attitude-orbit control of flexible solar sail for displaced solar orbit," Journal of Spacecraft and Rockets, vol. 50, no. 3, pp. 675-685, 2013.

[12] Y. Tsuda, T. Saiki, and R. Funase, "Generalized attitude model for spinning solar sail spacecraft," Journal of Guidance, Control, and Dynamics, vol. 36, pp. 967-974, 2013.

[13] R. L. Forward, "Solar photon thrustor," Journal of Spacecraft and Rockets, vol. 27, no. 4, pp. 411-416, 1990.

[14] C. R. McInnes, "Payload mass fractions for minimum-time trajectories of flat and compound solar sails," Journal of Guidance, Control, and Dynamics, vol. 23, no. 6, pp. 1076-1078, 2000.

[15] G. Mengali and A. A. Quarta, "Time-optimal threedimensional trajectories for solar photon thruster spacecraft," Journal of Spacecraft and Rockets, vol. 42, no. 2, pp. 379-381, 2005.

[16] A. D. Guerman and G. Smirnov, "Attitude control of solar photon thruster vs plane solar sail," Advances in the Astronautical Sciences, vol. 123, pp. 2635-22648, 2006.

[17] A. Guerman and G. Smirnov, "Attitude dynamics of a compound solar sail," in Proceedings of the AIAA 57th International Astronautical Congress (IAC '06), pp. 4090-4100, Valencia, Spain, October 2006.

[18] A. D. Guerman and G. Smirnov, "Compound solar sail with optical properties: models and performance," Journal of Spacecraft and Rockets, vol. 44, no. 3, pp. 732-734, 2007.

[19] A. D. Guerman, G. V. Smirnov, and M. C. Pereira, "Comparison of two compound solar sail schemes," in Proceedings of the International Conference on Numerical Analysis and Applied Mathematics (ICNAAM '10), vol. 1281, pp. 485-488, September 2010.

[20] B. Dachwald and P. Wurm, "Design concept and modeling of an advanced solar photon thruster," Advances in the Astronautical Sciences, vol. 134, pp. 723-740, 2009.

[21] B. Dachwald and P. Wurm, "Mission analysis and performance comparison for an advanced solar photon thruster," Advances in Space Research, vol. 48, no. 11, pp. 1858-1868, 2011.

[22] A. D. Guerman, G. V. Smirnov, and M. C. Pereira, "Orbital dynamics of a simple solar photon thruster," Mathematical Problems in Engineering, vol. 2009, Article ID 537256, 11 pages, 2009. 


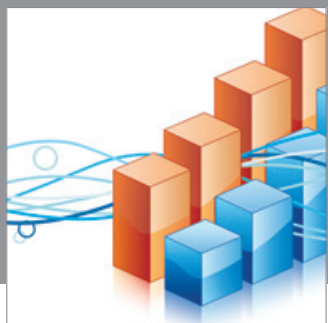

Advances in

Operations Research

mansans

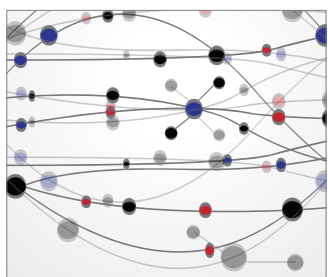

The Scientific World Journal
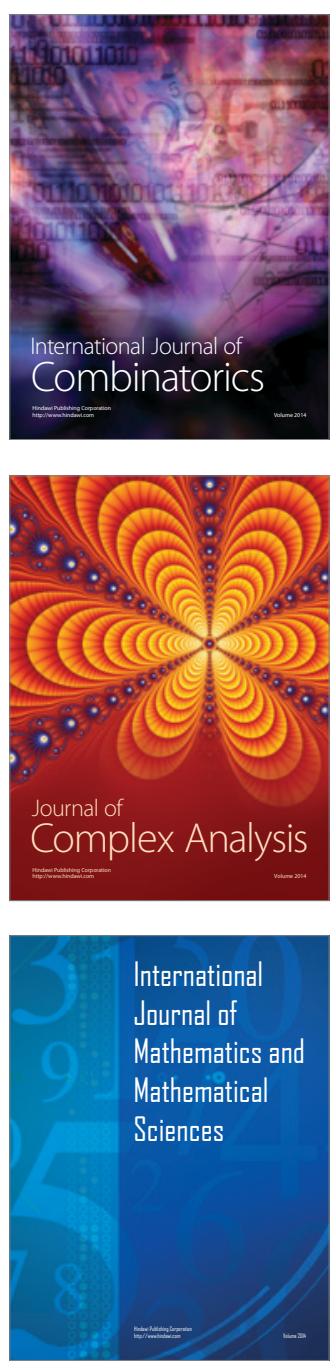
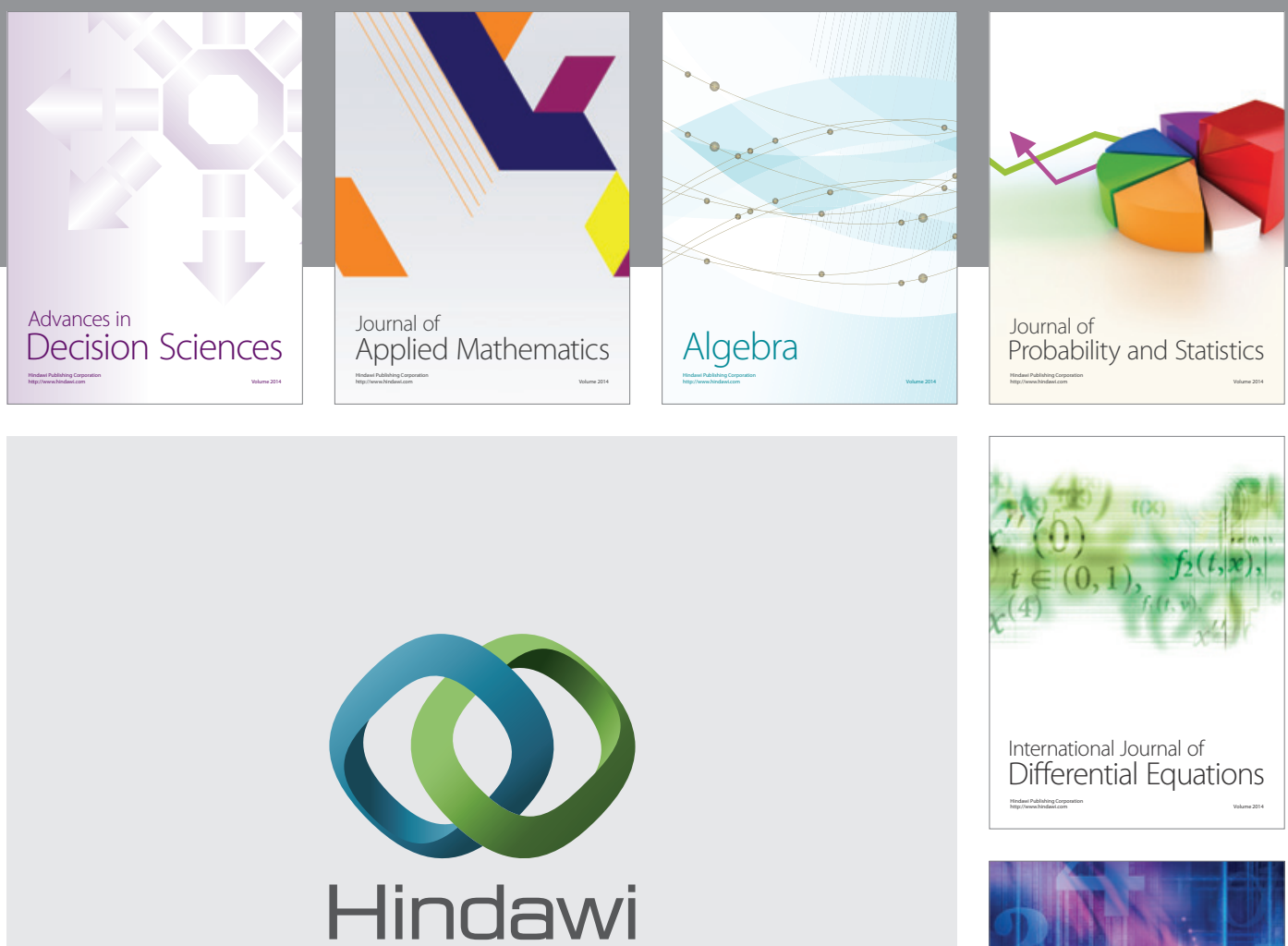

Submit your manuscripts at http://www.hindawi.com
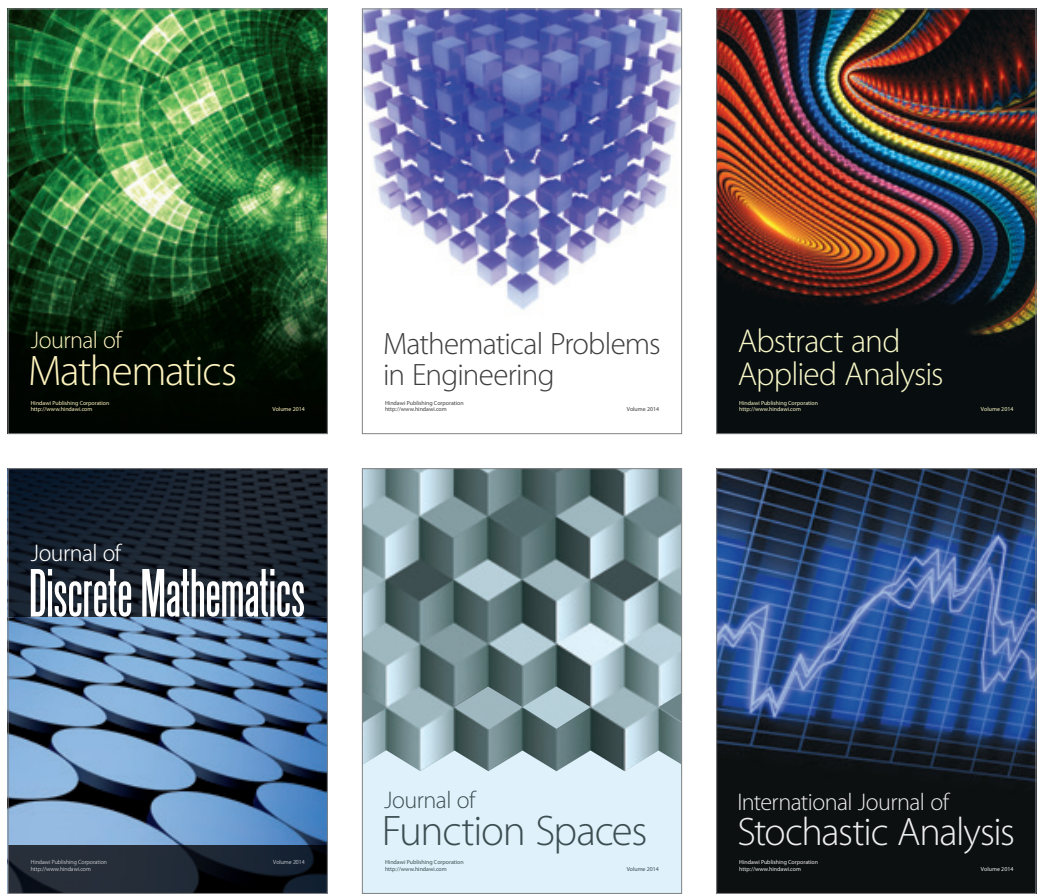

Journal of

Function Spaces

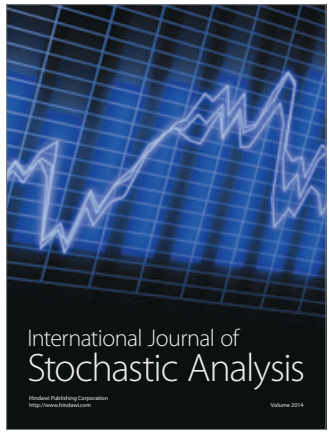

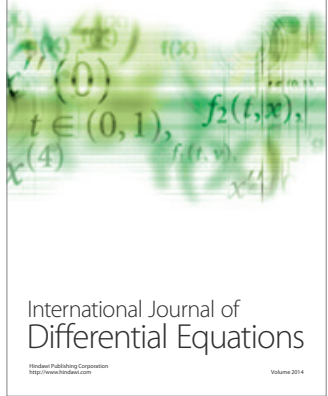
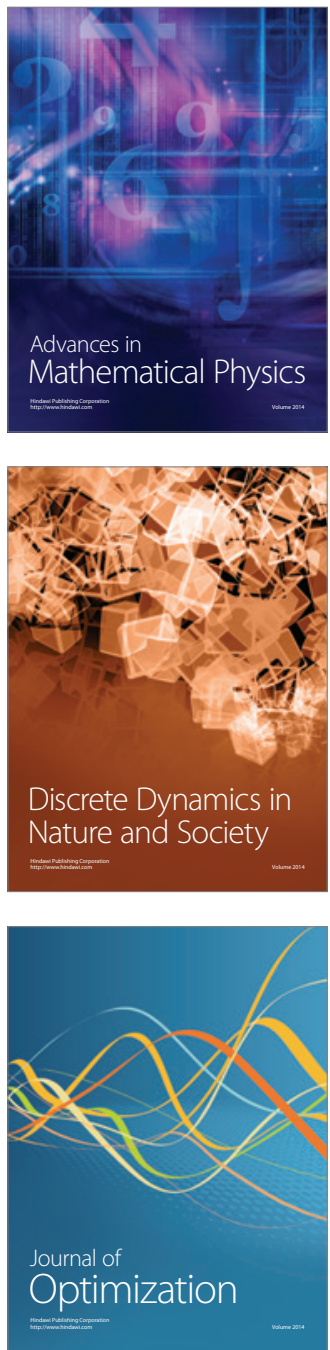